\title{
Target charging effects on proton acceleration during high-intensity short-pulse laser-solid interactions
}

\author{
F. N. Beg, ${ }^{\text {a) }}$ M. S. Wei, A. E. Dangor, A. Gopal, M. Tatarakis, and K. Krushelnick ${ }^{\text {b) }}$ \\ The Blackett Laboratory, Imperial College, London SW7 2BZ, United Kingdom \\ P. Gibbon \\ Research Centre Jülich GmbH, ZAM, D-52425 Jülich, Germany
}

E. L. Clark and R. G. Evans

AWE plc, Aldermaston, Reading RG7 4PR, United Kingdom

K. L. Lancaster and P. A. Norreys

Rutherford Appleton Laboratory, Chilton, Oxon, OX11 OQX, United Kingdom

K. W. D. Ledingham and P. McKenna

Department of Physics, University of Strathclyde, Glasgow G4 ONG, United Kingdom

M. Zepf

Department of Physics, Queen's University, Belfast BT7 1NN, United Kingdom

(Received 6 November 2003; accepted 27 January 2004)

We report results from experiments performed at the Rutherford Appleton Laboratory using the VULCAN laser facility $\left(I>5 \times 10^{19} \mathrm{~W} \mathrm{~cm}^{-2}\right)$. Single wire targets were used, and on some shots additional objects were placed near the target. These were positioned so that they were not irradiated by the laser. Proton emission from single wire targets was observed as radially symmetric structures ("stripes") in both the forward and backward directions, and was due to plasma sheath acceleration around the wire. The presence of objects in the vicinity of the interaction had a significant effect on the angular emission pattern of protons from the primary target. Importantly, the secondary object was also observed to be a source of energetic proton emission. (C) 2004 American Institute of Physics. [DOI: 10.1063/1.1689748]

There has been considerable recent progress in the study of proton beams ${ }^{1,2}$ generated from high-intensity short-pulse laser-solid interactions. The interest in laser-generated proton beams is primarily due to a number of potentially important applications, such as the creation of tabletop accelerators, the production of medical radioisotopes, and for fast ignition in inertial confinement fusion. Most of these applications require highly collimated beams with little energy spread. It has been observed ${ }^{2,3}$ that variations in target material and configuration can significantly affect the proton angular emission and energy spectrum.

In this letter, we present observations of proton emission from wire targets in several configurations. We show that the presence of an additional wire in the vicinity of the target wire significantly affects the spatial structure of the proton emission because of the transient electrostatic potential on the primary target, and the consequent plasma formation on secondary objects. Such effects may impact the design of targets for applications of these proton beams for probing ${ }^{4}$ and fast ignition. ${ }^{5}$ The additional wire was also observed to be a source of protons because of plasma formation and field emission due to target charging effects.

These experiments were carried out with the Chirped Pulse Amplification beam line of the VULCAN Nd:Glass laser system at the Rutherford Appleton Laboratory. The laser wavelength was $1.054 \mu \mathrm{m}$, the pulse length was $0.9-1.3$ ps, and the energy on target was between 60 and $80 \mathrm{~J}$. The

\footnotetext{
${ }^{a)}$ Present address: MAE Dept., Univ. of California, San Diego.

${ }^{b)}$ Electronic mail: k.krushelnic@imperial.ac.uk
}

$20 \times 11 \mathrm{~cm}^{2}$ laser beam was focused with an off-axis parabolic mirror having a focal length of $60 \mathrm{~cm}$, which resulted in a spot size of $15 \mu \mathrm{m}$. The maximum intensity was 5 $\times 10^{19} \mathrm{~W} \mathrm{~cm}^{-2}$. The diameter of the wire target was $20 \mu \mathrm{m}$, so there was little leakage of the laser radiation around the wire during a shot. The length of the wires (copper) was either 3 or $5 \mathrm{~mm}$. The wire axis was perpendicular to the polarization plane of the laser. On some shots, an additional wire was placed in the vicinity of the target wire (at a distance of 100-300 $\mu \mathrm{m}$ ). This additional wire was positioned such that direct illumination from the laser was avoided.

The angular distribution and energy spectra of the accelerated protons were measured using passive detector stacks consisting of alternate layers of radiochromic film (RCF) and CR39 plastic nuclear track detectors. These stacks were covered with $12 \mu \mathrm{m}$ thick aluminum foil to absorb low energy charged particles and plasma radiation. The stopping power of protons in RCF and CR39 is well known, so at a particular layer in the stack, the energy of protons producing the signal can be easily determined. The CR39/RCF detector stack was placed behind the target at $45^{\circ}$ to the main beam. A stack was also placed in the blowoff (illumination) direction for some shots.

Figure 1 displays typical scanned images of RCF and CR39 at different depths in the stack from an interaction with a copper wire. The stack (50 mm wide) was placed at $20 \mathrm{~mm}$ from the target covering a large angle in the forward direction $\left(100^{\circ}\right)$. In this case, the laser was incident near the top of 


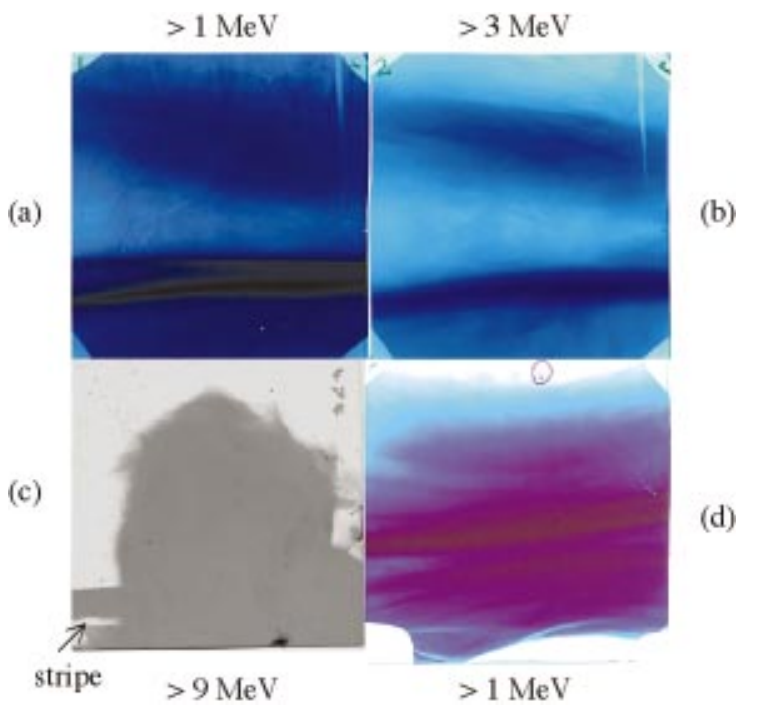

FIG. 1. (Color) Scanned images of (a), and (b) RCF and (c) CR39. The diffuse pattern and stripe structure is evident only on the CR39 detector $(E>9 \mathrm{MeV})$. RCF image (d) shows the stripe structure when the stack was placed in the blowoff direction and the laser was incident in the middle of the wire.

the wire. On the first layer of RCF, which is sensitive to protons of energies greater than $1 \mathrm{MeV}$, an intense "stripe" (containing some structure) can be observed among some other more diffuse structures. This stripe is uniform along the entire width of the RCF. On the second layer of RCF (sensitive to protons with energies greater than $3 \mathrm{MeV}$ ), the single intense stripe is more prominent. CR39 detectors deep within the stack also show a single stripe. We infer maximum proton energies from these interactions of $9 \mathrm{MeV}$ and an angular vertical width of $3.6^{\circ}$. A very diffuse emission pattern was also recorded on both CR39 layers. The size of the diffuse pattern decreased for higher proton energies. The horizontal angular emission is $22^{\circ}$ for $9 \mathrm{MeV}$ protons. It is interesting to note that the observed structure is emitted at $45^{\circ}$ to the laser beam propagation direction. This is probably due to the direction of the electron beam, which creates the space charge that accelerates protons away from the target.

On some shots, the focal spot on the wire was moved 2 $\mathrm{mm}$ from the tip. The diffuse structure on the RCF and the diffuse pattern on CR39 are not seen. Therefore, these are very likely a result of the laser focused near the tip and fast electrons moving over the top of the wire. When the stack of $\mathrm{RCF} / \mathrm{CR} 39$ was moved to the blowoff direction, a horizontal stripe with a similar vertical half angle as in the forward direction was observed [Fig. 1(d)]. There is a significant component of the proton emission which is symmetric around the wire, since fast electrons from the interaction region move around the target (i.e., they are reflected by the electrostatic fields created by space charge). These electrons will form an electrostatic sheath around the wire, resulting in proton emission in the form of a disk.

Observations were also made with an additional wire close to the target wire. Both wires were of equal length and $250 \mu \mathrm{m}$ apart. The laser was focused near the center of the target wire. The RCF/CR39 stack (Fig. 2) is consistent with two separate sources of protons, in that two well-separated stripes are evident in the RCF detectors. Both sets of stripes have half angles of $4^{\circ}-5^{\circ}$, and are likely due to emissions Downloaded 21 Dec 2006 to 134.94.122.39. Redistribution subject

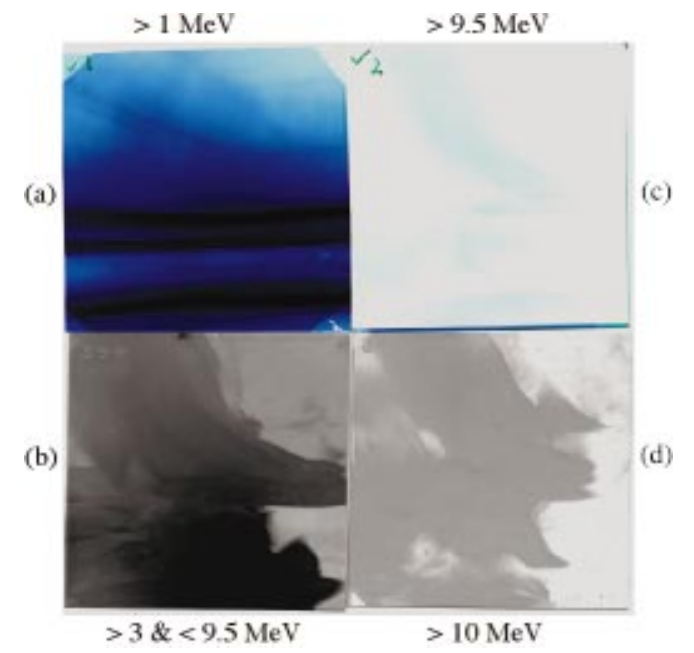

FIG. 2. (Color) (a) Scanned images of RCF which shows two sets of stripes due to protons $(>1 \mathrm{MeV})$. (b) A complex proton structure on the CR39 detector with proton energy between 3 and $9.5 \mathrm{MeV}$. Stripes are of low energy and are not seen in (c) RCF or (d) CR39.

from both the wire target and the additional wire. The separation between the stripes at $16^{\circ} \pm 8^{\circ}$ is probably due to a tilt between the wires, which was noted in this target before the laser shot, and which adds weight to the conclusion that the second stripe is indeed due to the proton emission from the secondary wire. On both CR39 detectors (Fig. 2), a complex emission pattern is observed, which is significantly different from that due to single wire targets. The stripe structure was recorded on the CR39 detector sensitive to protons above 3 $\mathrm{MeV}$, but was not observable on CR39 detectors sensitive to higher energy $(>9 \mathrm{MeV})$ protons. Similar well-separated, double-"stripe" emission patterns were observed when the stack was placed in the blowoff direction. The proton emission observed implies that hot electrons are generated from both wires as a result of target charging and field emission; even though only one of the wires was irradiated by the laser beam.

Single wire experiments were simulated using PEPC, a new gridless, electrostatic particle code which uses a parallel tree algorithm to compute interparticle potentials and forces directly in a time $O(N \log N)$. Technical details of this code are given in Ref. 6. As with conventional particle codes, the physical parameter space that a tree code can handle is also limited by computational cost. Therefore, we consider a $1 / 10$-scale wire model with radius $r_{w}=1 \mu \mathrm{m}$ and length 10 $\mu \mathrm{m}$. This is suspended in free space with open boundary conditions: particles are free to fly away from the interaction region and to circulate around the wire. The wire density is initially uniform with $n_{e}=n_{i}=4 n_{c}$, where $n_{c}$ is the critical density $\left(10^{21} \mathrm{~cm}^{-3}\right)$ due to computational limitations. A total of $1.4 \times 10^{6}$ electrons and ions were used, with the ions having an atomic number $Z=1$ and mass $m_{i} / m_{e}=1836$.

The laser is modeled as a ponderomotive standing wave potential (with both dc and oscillating components), initially focused near the midpoint of the wire with a $0.5 \mu \mathrm{m}$ FWHM $\sin ^{2}$ radial profile. To facilitate the computation, the intensity is ramped up over 10 fs to a peak value of 5 $\times 10^{19} \mathrm{~W} \mathrm{~cm}^{-2}$, held constant for $200 \mathrm{fs}$, and then switched AIP license or copyright, see http://apl.aip.org/apl/copyright.jsp 
Forward

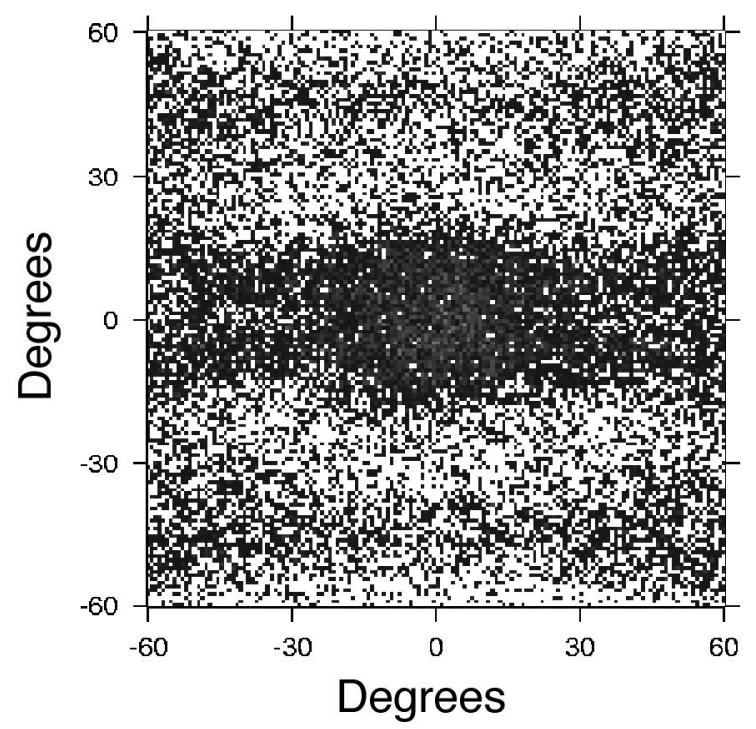

Backward

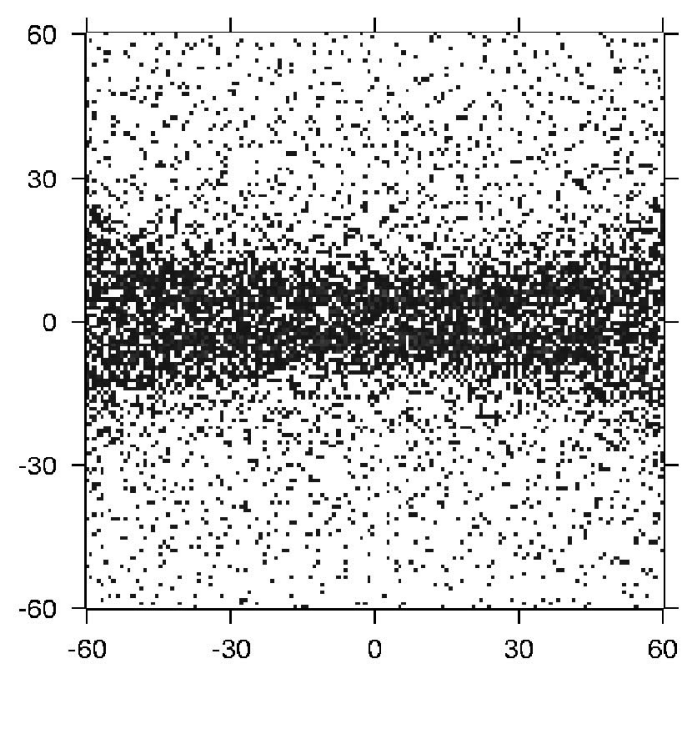

FIG. 3. Reconstruction of the far-field angular emission pattern (in degrees) of protons in forward and backward directions at 450 fs.

off. The turning point of the electromagnetic wave is assumed to be at the critical density surface, which is tracked inwards as it buckles under the laser pressure. The pulse duration is therefore restricted to values below $2 r_{w} / u_{h}$, where $u_{h}$ is the usual hole-boring velocity, ${ }^{7}$ in this case $0.05 c$ (where $c$ is the speed of light in vacuum).

That the target becomes highly positively charged as a result of the laser irradiation is supported by the simulation results. Apart from the region surrounding the hole, the target is uniformly charged to a potential of around $0.5 \mathrm{MV}$. The spatial extent of the potential "envelope" grows with time. At $160 \mathrm{fs}$, the electron cloud seen in the phase-space plot fills a region 30 times larger than the initial wire radius. Two types of ion emission, beam- and disk-like, are seen in the simulation. The first of these is produced along the laser axis via the ponderomotive shock. Monitoring the time development of the longitudinal ion phase space ( $p_{x}$ vs $x$ ) shows that, in this case, emission is dominated by front-side ions, which also exist the wire with more energy than the rearside, sheath-accelerated ions. The maximum energy in this forward-directed beamlet is $9 \mathrm{MeV}$, although the main component is at around $3 \mathrm{MeV}$.

The far-field emission pattern corresponding to the RCF and CR39 images is reconstructed in Fig. 3, which, for statistical reasons, displays the angular distribution of the transverse/longitudinal momenta $f(\alpha, \beta)$, where $\alpha$ $=\arctan \left(p_{y} / p_{x}\right)$ and $\beta=\arctan \left(p_{z} / p_{x}\right)$, rather than the particle count on a virtual collector plate. In both forward and backward directions, we observe the same double-stripe structure seen in Fig. 1, albeit at somewhat lower energies (0.5-1.5 $\mathrm{MeV}$ ). This structure continues to develop after the laser has been turned off, suggesting that it is influenced by return currents, which again depend on the absolute amount of hot electron charge initially stripped from the wire by the laser. ${ }^{8}$

In conclusion, protons from the wire targets are generated symmetrically around a wire when a high intensity laser is focused on the wire. If the focal spot is close to the tip of the wire, the proton structure is modified. We have clearly Downloaded 21 Dec 2006 to 134.94.122.39. Redistribution subject shown that the presence of additional wire in the vicinity of the wire targets affects proton emission significantly.

These results clearly indicate that objects in close proximity of the main target can themselves become additional sources of energetic protons because of plasma sheath formation on the secondary object (even though screened from direct laser irradiation). Consequently, such effects may have important implications for some of the proposed applications of these proton beams.

The authors would like to acknowledge assistance of the staff of the Central Laser Facility of the Rutherford Appleton Laboratory. We also thank the UK Engineering and Physical Sciences Research Council (EPSRC). P.G. acknowledges a grant of computing resources on the Jülich IBM p690+ cluster, project JZAM04.

\footnotetext{
${ }^{1}$ A. Pukhov, Rep. Prog. Phys. 66, 47 (2003).

${ }^{2}$ E. L. Clark, K. Krushelnick, J. R. Davies, M. Zepf, M. Tatarakis, F. N. Beg, A. Machacek, P. A. Norreys, M. I. K. Santala, I. Watts, and A. E. Dangor, Phys. Rev. Lett. 84, 670 (2000) R. A. Snavely, M. H. Key, S. P. Hatchett, T. E. Cowan, M. Roth, T. W. Phillips, M. A. Stoyer, E. A. Henry, T. C. Sangster, M. S. Singh, S. C. Wilks, A. MacKinnon, A. Offenberger, D. M. Pennington, K. Yasuike, A. B. Langdon, B. F. Lasinski, J. Johnson, M. D. Perry, and E. M. Campbell, ibid. 85, 2945 (2000); M. Zepf, E. L. Clark, F. N. Beg, R. J. Clarke, A. E. Dangor, A. Gopal, K. Krushelnick, P. A. Norreys, M. Tatarakis, U. Wagner, and M. S. Wei, ibid. 90, 064801 (2003).

${ }^{3}$ M. Roth, A. Blazevic, M. Geissel, T. Schlegel, T. E. Cowan, M. Allen, J.-C. Gauthier, P. Audebert, J. Fuchs, J. Meyer-ter-Vehn, M. Hegelich, S. Karsch, and A. Pukhov, Phys. Rev. ST Accel. Beams 5, 061301 (2002).

${ }^{4}$ M. Borghesi, A. Schiavi, D. H. Campbell, M. G. Haines, O. Willi, A. J. MacKinnon, L. A. Gizzi, M. Galimberti, R. J. Clarke, and H. Ruhl, Plasma Phys. Controlled Fusion 43, A267 (2001).

${ }^{5}$ M. Roth, T. E. Cowan, M. H. Key, S. P. Hatchett, C. Brown, W. Fountain, J. Johnson, D. M. Pennington, R. A. Snavely, S. C. Wilks, K. Yasuike, H. Ruhl, F. Pegoraro, S. V. Bulanov, E. M. Campbell, M. D. Perry, and H. Powell, Phys. Rev. Lett. 86, 436 (2001).

${ }^{6}$ P. Gibbon, PEPC: Pretty Efficient Parallel Coulomb Solver, ZAM Technical Report FZJ-ZAM-IB-2003-05 (2003), available online at http:// www.fz-juelich.de/zam/docs/autoren2003/gibbon.html

${ }^{7}$ S. C. Wilks, W. L. Kruer, M. Tabak, and A. B. Langdon, Phys. Rev. Lett. 69, 1383 (1992).

${ }^{8} \mathrm{P}$. Gibbon (unpublished).
} 